\title{
INTERNAL ROTATION DISLOCATION OF THE SHOULDER
}

\author{
Report of a Case
}

\author{
L. S. Michaelis, London, England
}

Dislocations of the shoulder other than anterior dislocations are uncommon: complete posterior dislocations, for instance, account for probably no more than 1 per cent. of all cases, but-being readily shown by routine radiography-are unlikely to be overlooked. Less obvious displacement of the humeral head does occur, however, and diagnosis is liable to be missed. Such cases have sometimes been classified as posterior subluxations. They may present serious difficulties because the opportunity for straightforward manipulative reduction is lost and the hazards of late manipulation and open reduction (Watson-Jones 1943) have to be faced. The following case report illustrates not only the diagnostic and therapeutic difficulties associated with this type of injury, but also the need for defining more clearly the exact nature of the displacement. Open reduction provided an opportunity of determining accurately the nature of the pathology.

\section{CASE REPORT}

A woman aged fifty-nine, healthy and unusually muscular, fell from her autocycle on to the outstretched left upper limb. There was immediate severe pain in the left shoulder and no movement of the arm was possible. Antero-posterior and lateral radiographs taken within a few hours of the injury were reported as showing nothing abnormal. Her doctor was not satisfied and sent her to another hospital. Further radiographs, including stereoscopic films, failed to show any abnormality. A diagnosis of severe "sprain" was made. Four weeks later pain and limitation of movement were still present and the patient was referred to the author.

On examination, the left humerus was found to be held in maximal adduction and internal rotation. The head could not be palpated either in its normal place or anteriorly. Nor was it present in one of the two typical positions of posterior dislocation. The powerful deltoid muscle and remnants of the post-traumatic swelling made palpation uncertain. No movement of the shoulder was possible; fixed adduction was so extreme that pressure of the elbow had left a red mark on the skin of the patient's flank. There were no signs of vessel or nerve involvement. Examination under an anaesthetic confirmed that the shoulder was still firmly locked. Some scapular movement was possible, but no true shoulder movement. A gentle attempt at manipulation was made. While traction was applied the inward rotation was further increased. The joint unlocked with an audible snap and about half the normal range of external rotation and abduction became possible. It was foreseen that redisplacement might occur and in spite of precautionary measures this in fact happened within forty-eight hours. Locking was once more complete. Open reduction was planned.

Operation was undertaken five weeks after the accident. The shoulder was exposed through Bankart's anterior approach. The subscapularis tendon was found to be stippled with old haemorrhages and adherent to the capsule. The capsule, the front of which was thickened and rather slack, was incised. It was then seen that the greater tuberosity of the humerus was directed forwards, and that the neck of the humerus faced the glenoid. The articular surface of the head of the humerus could not be seen; the head, rotated inwards through nearly 90 degrees, was clearly locked at the posterior rim of the glenoid. Reduction was 
achieved by strong traction and lateral rotation. Multiple adhesions to the posterior part of the capsule were divided. The wound was closed in layers. The arm was immobilised in abduction and outward rotation for a few days, after which exercises were encouraged. The post-operative course was complicated by a small pulmonary infarct, possibly derived from the axillary vein, but a useful range of painless shoulder movement was regained within four weeks of operation. One year later the patient wrote to say that movement had increased further, and that the shoulder was painless. Power was good; she was able to do her hair, and was undertaking heavy housework and farm work.

\section{DISCUSSION}

In this case the doctor, rightly suspecting dislocation on clinical grounds, was misled by the inability of radiological methods to establish the diagnosis. The shortcomings of the routine methods of radiography of the shoulder have been discussed by Warrick (1948), who recommended that a vertical view should be taken with either the tube or a curved cassette placed in the axilla. His illustrations of three cases show clearly a posterior displacement of the head of the humerus. In his opinion (personal communication) two of his cases were identical with that described here. But in Warrick's cases it was possible to obtain sufficient abduction of the arm to place either the tube or the film beneath the axilla: it is therefore a matter of doubt whether they were really identical. In the present case no abduction was possible and it was impracticable to obtain the views recommended by Warrick. One of the radiographs of the writer's patient shows the state of extreme inward rotation (Fig. 1), but before operation it was incorrectly interpreted.

The difference between internal rotation dislocation and the usual type of posterior

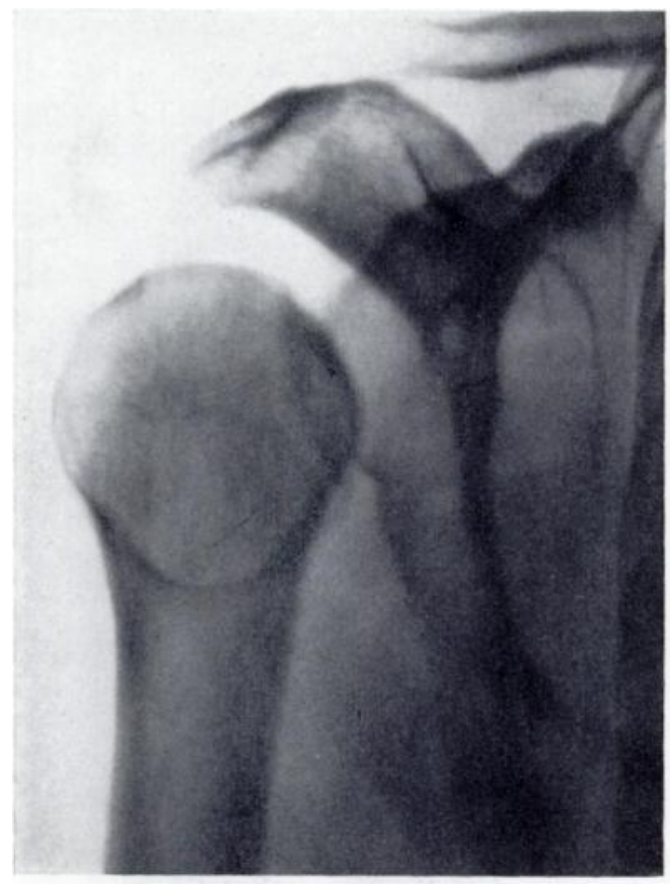

FIG. 1

Internal rotation dislocation of the shoulder. Radiograph before operation showed extreme internal rotation of the humerus, but the significance of this was not at first appreciated. 


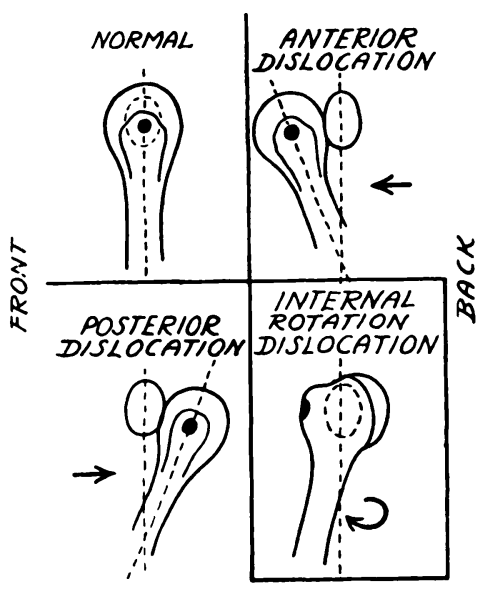

Fig. 2

Lateral aspect diagrams of left shoulder, showing the difference between common types of anterior and posterior dislocations and internal rotation dislocation. In the latter the front of the neck of the humerus is in contact with the glenoid fossa.

dislocation is illustrated in Figure 2, which shows the shoulder as seen from the lateral aspect; the most lateral point of the greater tuberosity is indicated by a black spot. This is directed laterally in the normal position and in the usual types of anterior and posterior dislocation. In internal rotation dislocation it is directed anteriorly. It will be noted further that in anterior and posterior dislocations the glenoid is not covered by the upper end of the humerus, which is displaced respectively forwards and backwards. But in internal rotation dislocation the upper end of the humerus maintains a relationship to the glenoid, not by its articular surface as in the normal shoulder, but by the anterior aspect of the neck. Early diagnosis alone can ensure early reduction by manipulation, which in the first few days should present little difficulty. Since radiography cannot always be relied upon to provide clear evidence of displacement, diagnosis should be based on the clinical findings of locking of the limb in adduction and inward rotation.

\section{REFERENCES}

WARrick, C. K. (1948): Posterior dislocation of the shoulder joint. Journal of Bone and Joint Surgery, 30-B, 651 .

Watson-Jones, R. (1943): Fractures and Joint Injuries. Edinburgh : E. \& S. Livingstone Ltd.

vol. $32 \mathrm{~B}$, No. 2 , MAY 1950 